\title{
Potential of Resveratrol Analogues as Antagonists of Osteoclasts and Promoters of Osteoblasts
}

\author{
Katarzyna Kupisiewicz • Patrice Boissy $\cdot$ Basem M. Abdallah • \\ Frederik Dagnaes Hansen • Reinhold G. Erben · Jean-Francois Savouret • \\ Kent Søe • Thomas L. Andersen • Torben Plesner • Jean-Marie Delaisse
}

Received: 8 October 2009/Accepted: 11 June 2010/Published online: 15 September 2010

(C) The Author(s) 2010. This article is published with open access at Springerlink.com

\begin{abstract}
The plant phytoalexin resveratrol was previously demonstrated to inhibit the differentiation and bone resorbing activity of osteoclasts, to promote the formation of osteoblasts from mesenchymal precursors in cultures, and inhibit myeloma cell proliferation, when used at high concentrations. In the current study, we screened five structurally modified resveratrol analogues for their ability to modify the differentiation of osteoclasts and osteoblasts
\end{abstract}

The authors have stated that they have no conflict of interest.

Electronic supplementary material The online version of this article (doi:10.1007/s00223-010-9399-3) contains supplementary material, which is available to authorized users.

K. Kupisiewicz $(\bowtie) \cdot$ P. Boissy · K. Søe .

T. L. Andersen - T. Plesner - J.-M. Delaisse

Division of Hematology, Department of Clinical Cell Biology,

Vejle Hospital, IRS/CSFU, University of Southern Denmark,

Vejle, Denmark

e-mail: kkupisiewicz@health.sdu.dk

B. M. Abdallah

Molecular Endocrinology Laboratory (KMEB),

Department of Endocrinology, Odense University Hospital,

Medical Biotechnology Center, University of Southern

Denmark, Odense, Denmark

F. D. Hansen

Department of Medical Microbiology and Immunology,

University of Aarhus, Aarhus, Denmark

R. G. Erben

Department of Biomedical Sciences,

University of Veterinary Medicine, Vienna, Austria

J.-F. Savouret

Pharmacology, Toxicology and Cell Signalization,

Cartilage Signaling and Pharmacology,

Université Paris Descartes, Paris, France and proliferation of myeloma cells. Compared to resveratrol, analogues showed an up to 5,000-fold increased potency to inhibit osteoclast differentiation. To a lesser extent, resveratrol analogues also promoted osteoblast maturation. However, they did not antagonize the proliferation of myeloma cells. The potency of the best-performing candidate in vitro was tested in vivo in an ovariectomy-induced model of osteoporosis, but an effect on bone loss could not be detected. Based on their powerful antiresorptive activity in vitro, resveratrol analogues might be attractive modulators of bone remodeling. However, further studies are required to establish their efficacy in vivo.

Keywords Resveratrol - Resveratrol analogue .

Osteoclast · Osteoblast $\cdot$ Multiple myeloma

Resveratrol (RSV, trans-3,5,4'-hydroxystilbene) is a naturally occurring phytoalexin generated in a number of plants including grapes, mulberries, cranberries, and peanuts. RSV has recently attracted considerable interest because of its inhibitory activity on multiple cellular events associated with carcinogenesis [1], and its actions on a broad cell spectrum are well documented both in vitro and in vivo [2, 3]. These anti-cancer effects of RSV are supported by observations in different cancer cell lines in vitro and in animal cancer models such as breast cancer [4], skin cancer [1], liver cancer [5], colorectal and intestinal cancers [6, 7], lung cancers [8], and neuroblastoma [9]. The results of preclinical studies have stimulated an interest from the pharmaceutical industry, and several clinical studies are ongoing in the field of cancer (colon cancer, non-Hodgkin lymphoma, and multiple myeloma) and other areas of clinical research. 
It was also reported that RSV may have beneficial effects on osteoclasts (OCs) and osteoblasts (OBs) [10-13]. Noteworthy, current therapies act mainly on only one of the remodeling processes either by inhibition of OC-mediated bone resorption (e.g., bisphosphonates) or by stimulation of bone formation by OBs (e.g., PTH or teraparatide) [14]. It is thus of interest that RSV has the potential both to antagonize OCs and to promote OBs. To our knowledge, only strontium ranelate, statins, and bortezomib share the same potential. Furthermore, RSV and the latter two drugs also prevent the proliferation of cancer cells in multiple myeloma [10, 15, 16]. Multiple myeloma is an incurable neoplasia of terminally differentiated B cells (plasma cells) that frequently causes extensive osteolytic bone lesions resulting in pathological fractures of bone, vertebral collapse, pain and disability. The bone lesions result from a combination of increased bone resorption and inhibition of bone formation in the vicinity of myeloma cells [17]. It thus appears that RSV possesses an ideal triad of properties: it suppresses OC differentiation while at the same time stimulating the differentiation of OBs and inhibits myeloma cell proliferation and survival [10].

However, these effects on OCs, OBs, and myeloma cells were seen only when RSV was used at concentrations between 50 and $100 \mu \mathrm{M}$, while lower concentrations seem to be ineffective. In both humans and rodents the peak plasma levels of unmetabolized RSV are well below this level even after a high oral dose, and its elimination is rather rapid [18, 19]. Another problem is that the high doses of RSV that are sufficient to obtain the desired plasma concentrations can cause side effects such as renal toxicity seen in rodents [20]. Furthermore, taking into account the broad spectrum of cellular actions [reviewed in 2, 3], RSV is a questionable option for tailored therapies where a precise effect is needed. A particular concern is its estrogen receptor (ER) agonistic activity [21] associated with genotoxicity [22] and increased risk of carcinogenesis.

Given the limited bioavailability of RSV after oral administration, the high concentrations required to obtain the desired effects seen in vitro, its potential toxicity, and its complex mechanisms of actions, RSV analogues may offer advantages over the parent compound. Structural modifications of RSV led to analogues with improved potency and specificity compared with the parental agent, thereby being more tolerable and safer [23, 24]. One of the RSV derivatives (STR501) is currently being tested in a phase II clinical trial for the treatment of multiple myeloma as a potent activator of Silent Information Regulator T-1, controlling bone mass in vivo via direct regulation of $\mathrm{OBs}$ and OCs [25].

In the current study, we compared the activity of a set of RSV derivatives with the parental product. They were designed for no affinity for the ER and higher affinity toward the aryl hydrocarbon receptor (AhR). The modifications in structure consisted of chloride substitution, contributing to suppress ER affinity and to increase AhR affinity [26]. ER $\alpha$ and $\operatorname{ER} \beta$ (jointly referred to as "ER" in this article) are activated upon the ligation of estrogen. ER functions mainly as a DNA-binding transcription factor that regulates gene expression [27]. The engagement of ER was shown to antagonize OCs and inhibit bone resorption [28]. AhR is a transcription factor that mediates the toxicity of ubiquitous environmental contaminants. AhR ligands are prevalent in tobacco smoke [29], pesticides, and some manufactured chemicals [30]; and they may elicit toxic responses such as tumour promotion [31] and immunosuppression [32]. Emerging data indicate that AhR may also play a role in bone metabolism, mainly acting on $\mathrm{OB}$ maturation and the modulation of OB functions [33]. Therefore, novel RSV derivatives with restricted activity toward ER or AhR may serve as unique tools to determine whether the effects of the parental compound RSV are due to one or the other pathway.

In the present study we tested the activity of five of these RSV analogues on OCs, OBs, and myeloma cells in cultures. The best candidate was selected for in vivo testing, using ovariectomized (OVX) rats as a model of estrogeninduced bone loss.

\section{Materials and Methods}

\section{RSV Analogues}

RSV derivatives displaying different affinity toward ER and AhR are shown in Table 1 and further described in de Medina et al. [26]. Additional PDM02, PDM10, and PDM11 stocks were supplied from Cayman Chemical (Tallinn, Estonia) and solubilized in sterile 99\% ethanol. For the animal study PDM02 was dissolved in DMSO.

\section{Cell Cultures}

Myeloma cell lines U266 and OPM-2 were obtained from DSMZ (Braunschweig, Germany) and cultured at $37^{\circ} \mathrm{C}$ in RPMI 1640 medium (GIBCO, Taastrup, Denmark) supplemented with $10 \%$ FCS. Media were replaced every other day. Primary myeloma cells were isolated from bone marrow aspirates from myeloma patients as a part of the diagnostic procedure after informed consent (ethical committee approval S-20070019). Myeloma cells were sorted immunomagnetically using MACS anti-CD138 magnetic beads (Miltenyi Biotech, Bergisch Gladbach, Germany) as recommended by the manufacturer. The purity of isolated 
Table 1 Receptor binding and biological activity of RSV analogues used in the study

\begin{tabular}{|c|c|c|c|c|c|c|}
\hline \multirow[t]{2}{*}{ Compound } & \multirow[t]{2}{*}{$\mathrm{R}_{1}$} & \multirow[t]{2}{*}{$\mathrm{R}_{2}$} & \multicolumn{2}{|l|}{ AhR } & \multicolumn{2}{|l|}{ ER } \\
\hline & & & $\mathrm{K}_{\mathrm{i}} \mathrm{nM}$ & Activity & $\mathrm{K}_{\mathrm{i}} \mathrm{nM}$ & Activity \\
\hline RSV & $4^{\prime}-\mathrm{OH}$ & $\mathrm{OH}$ & $169 \pm 5.2$ & Antagonist & $785 \pm 4.1$ & Agonist \\
\hline PDM01 & $4^{\prime}-\mathrm{OMe}$ & $\mathrm{OMe}$ & $7.7 \pm 0.2$ & Antagonist & $261 \pm 3.2$ & Agonist \\
\hline PDM02 & $4^{\prime}-\mathrm{Cl}$ & CI & $1.2 \pm 0.4$ & Antagonist & $>100,000$ & \\
\hline PDM06 & $4^{\prime}-\mathrm{CF}_{3}$ & $\mathrm{CF}_{3}$ & $2.1 \pm 0.8$ & Antagonist & $>100,000$ & \\
\hline PDM10 & $4^{\prime}-\mathrm{CF}_{3}$ & $\mathrm{CI}$ & $0.2 \pm 0.4$ & Agonist & $>100,000$ & \\
\hline PDM11 & $4^{\prime}-\mathrm{OMe}$ & $\mathrm{CI}$ & $1.4 \pm 0.7$ & Antagonist & $>100,000$ & \\
\hline
\end{tabular}

Modified from de Medina et al. [26] with permission of the author

$A h R$ aryl hydrocarbon receptor, $E R$ estrogen receptor

myeloma cells varied from 70 to $90 \%$ as determined by flow cytometric analysis with anti-CD138 antibody (Becton Dickinson, Heidelberg, Germany).

To monitor cell viability upon treatment with RSV analogues, myeloma cells were seeded in flat-bottomed 96well plates at $5 \times 10^{4}$ cells/well in $200 \mu \mathrm{L}$ of RPMI 1640 medium in the presence or absence of $10 \%$ FCS and increasing concentrations of RSV analogues. The metabolic activity of the cells as a surrogate marker of cell viability was measured with the XTT Cell Proliferation Kit (Roche, Hvidovre, Denmark) according to the manufacturer's recommendations. Apoptotic cells were labeled with Annexin V and propidium iodide (Annexin-V Apoptosis Detection Kit, BD Pharmingen, Brondby, Denmark) and analyzed on a flow cytometer (Becton Dickinson).

Human OC precursors were prepared from whole blood obtained from healthy donors at Vejle Hospital after written informed consent (ethical committee approval $\mathrm{S}-20070019)$. Isolation of $\mathrm{CD} 14^{+}$monocytes, OC differentiation, and monitoring of both tartrate-resistant acid phosphatase (TRAP) activity and number of TRAP-positive multinucleated cells were performed as described previously [10]. Cell viability upon treatment with RSV analogues was determined using Cell Titer Blue Viability Assay (Promega, Nacka, Sweden) according to the manufacturer's recommendations.

For OB differentiation, we used the well-characterized human bone marrow mesenchymal stem cell line hMSCTERT (hMSC), which stably expresses human telomerase reverse transcriptase gene (TERT) [34, 35]. hMSC cells were grown in phenol red-free MEM (GIBCO) supplemented with $10 \%$ FCS. At $60-70 \%$ cell confluence, media were replaced with $\mathrm{OB}$ differentiating medium (ODM) containing $10^{-9} \mathrm{~mol} / \mathrm{L} 1,25(\mathrm{OH})_{2} \mathrm{D}_{3}, 50 \mu \mathrm{g} / \mathrm{mL}$ L-ascorbic acid, $10^{-9} \mathrm{~mol} / \mathrm{L}$ dexamethasone, and $10^{-3} \mathrm{~mol} / \mathrm{L} \quad \beta$ glycerophosphate (all from Sigma, Brondby, Denmark) and increasing concentrations of RSV analogues. hMSC-TERT cells were then cultured at $37^{\circ} \mathrm{C}$ up to 5 days, with media replacement every other day. Cell viability upon treatment with RSV analogues was assessed using the Cell Titer Blue Viability Assay (Promega) as recommended by the manufacturer.

\section{Bone Resorption Assays}

For bone resorption assays [10], OC precursors were cultured for 2 days with rhM-CSF $(25 \mathrm{ng} / \mathrm{mL})$ and thereafter differentiated for 7 days with rhM-CSF and rhRANKL (25 ng/mL each; R\&D Systems, Abingdon, UK). Cells were harvested by trypsin treatment and seeded on bone slices (6 $\mathrm{mm}$ in diameter; IDS, Herlev, Denmark) at a density of $10^{5}$ cells in $200 \mu \mathrm{L}$ of $\alpha$-MEM supplemented with $10 \%$ FCS, rhM-CSF, rhRANKL (25 ng/mL each), and increasing concentrations of RSV analogues. OCs were cultured at $37^{\circ} \mathrm{C}$ for up to 3 days. At the end of the experiment, cells were scraped off in water using a cotton stick, and resorption pits were visualized with $0.1 \%$ toluidine blue staining. The resorbed areas on bone slices were quantified using an upright light microscope (Carl Zeiss, Birkeroed, Denmark) equipped with a 36-point grid, and results were presented as the total resorbed area in percentage of the whole bone surface.

\section{Real-Time Quantitative PCR on OC Cultures}

Relative gene expression was analyzed by TaqMan quantitative real-time PCR as described previously [10] using primers and probes for cathepsin K (Hs00355885_m1), 
RANK (Hs00187189_m1), c-fms (Hs00234617_m1), and CD14 (Hs00169122_q1). NFATc1 primers and probe were designed according to the NFATc1 Genbank accession U08015: NFATc1-fp TGGAGAGTCCTCGCATCGA, NFATc1-rp CATCGTGGAAAAACTGGTTATTGT, and NFATc1-p ATAACCTCGTGCTTGGGCCTGTACCAC. All primers and probes were purchased from Applied Biosystems (Naerum, Denmark). Cultures of differentiating OCs treated with RSV analogues were harvested at the time points indicated in figure legends, lysed, and subjected to RNA isolation. Residual cell debris was removed by centrifugation through QIA Shredder columns (Qiagen, Hilden, Germany). Total RNA was purified from the cleared homogenate using the RNeasy Mini kit (Qiagen). The yield and purity of isolated RNA were verified by spectrophotometry before reverse-transcribing into singlestranded cDNA using the Iscript cDNA Synthesis kit (BioRad Laboratories, Copenhagen, Denmark) as recommended by the manufacturer. The relative standard curve method was used to calculate the amplification difference for each primer set. A standard curve was made from six points corresponding to twofold cDNA serial dilutions for each gene. For each sample, the relative amount was calculated from its respective standard curve. The quantity of the target gene mRNA was then obtained by division of each value by the mean relative gene expression of human GUS (36-3026) and ABL (06-1250) used as endogenous controls [10].

\section{In Situ Detection of NF- $\kappa$ B Activation}

For in situ detection of nuclear factor- $\kappa \mathrm{B}$ (NF- $\kappa \mathrm{B})$ translocation, isolated $\mathrm{CD} 14^{+}$cells were seeded in chambered slides and treated for 2 days with $25 \mathrm{ng} / \mathrm{mL}$ rhM-CSF. Thereafter, cells were incubated in the presence or absence of increasing concentrations of RSV analogue PDM02 or $\mathrm{RSV}$ at $100 \mu \mathrm{M}$ for $1 \mathrm{~h}$ or 7 days in $\alpha \mathrm{MEM}$ containing $10 \%$ FCS and $25 \mathrm{ng} / \mathrm{mL}$ rhM-CSF and then exposed to $100 \mathrm{ng} / \mathrm{mL}$ of rhRANKL for $30 \mathrm{~min}$ at $37^{\circ} \mathrm{C}$. Cells were then fixed and stained with mouse monoclonal anti-p65 antibody as described [10]. Cells showing bright staining for p65 in the nucleus were scored, and the results were presented as the number of cells with NF- $\kappa \mathrm{B}$ nuclear translocation as a percentage of the total number of cells counted.

\section{Real-Rime Quantitative PCR on OB Cultures}

For real-time PCR analysis of the expression of OB markers, total RNA was isolated from cultured hMSCTERT cells using a single-step method with TRIzol (Invitrogen, Taastrup, Denmark) according to the manufacturer's instructions. The integrity and purity of isolated
RNA were verified by spectrophotometry and gel electrophoresis, and total RNA was then transcribed into cDNA using RevertAid H Minus first-strand cDNA synthesis kit (Fermentas, Copenhagen, Denmark) as recommended by the manufacturer. Quantitative PCR and gene expression analysis of the target gene and endogenous control $\beta$-actin was done as described previously [36]. Expression levels for each target gene and endogenous control were calculated using the comparative $C_{t}$ method $\left[\left(1 /\left(2^{\Delta C t}\right)\right]\right.$ formula.

\section{Animal Study}

Sixty female virgin 8-month-old Sprague-Dawley rats (Taconic, Lille Skensved, Denmark) were maintained under standard conditions of 12-h day and night cycles. Rats were housed two per cage and received common chow and water ad libitum. Handling of the rats was in full accordance with the European Community guidelines for the care and management of laboratory animals.

Rats were randomized into six groups ( $n=10$ /group), (1) sham-operated (Sham), (2) OVX treated with estrogen (OVX + estrogen), (3) OVX treated with vehicle (OVX + DMSO), (4) untreated OVX, and OVX rats treated with (5) high (40 mg/kg) or (6) low (4 mg/kg) dose of PDM02. At surgery, 60-day slow-release pellets containing $0.05 \mathrm{mg}$ $17 \alpha$-ethylenestradiol (Innovative Research of America, Sarasota, FL) were implanted subcutaneously in the OVX + estrogen group. For vehicle or PDM02 administration, rats were deprived of food for $8 \mathrm{~h}$, anesthetized with $4 \%$ isoflurane, and injected intraperitoneally every other day.

The body weight and health status of the animals were monitored weekly. Blood samples (eye puncture) were taken before surgery, twice during treatment (weeks 2 and 4), and at the end of the experiment (week 6, cardiac puncture). Rats were killed by an overdose of pentobarbital, and the right and left tibiae were collected for further analysis.

Volumetric BMD of tibiae was measured by peripheral quantitative computed tomography (pQCT) using the XCT Research M+ pQCT machine (Stratec Medizintechnik, Pforzheim, Germany). The voxel size was $0.100 \mathrm{~mm}$. One slice in the mid-diaphysis of the tibiae located $2 \mathrm{~mm}$ proximal to the tibia-fibular junction and one slice in the proximal tibial metaphysis located $2 \mathrm{~mm}$ distal from the growth plate were measured. A threshold of $710 \mathrm{mg} / \mathrm{cm}^{3}$ was used for calculation of cortical BMD. For the measurement of trabecular BMD in the proximal tibial metaphysis, we used contour mode 1 and peel mode 20 . The percentage of trabecular bone was set to $50 \%$ of the cross-sectional area.

Serum levels of CTX, a bone resorption marker, were measured using Rat CrossLaps (IDS) as recommended by the manufacturer. 


\section{Statistical Analysis}

Statistical comparisons were done with Student's $t$-test or one-way ANOVA followed by Dunnett's post-test, and $P \leq 0.05$ was considered significant (Prism v.5 program; GraphPad, San Diego, CA). EC 50 values were obtained using nonlinear regression of the original data.

\section{Results}

Effect of RSV Analogues on Differentiating and Mature OCs

Since RSV was previously shown to suppress RANKLmediated OC differentiation [10], we examined whether RSV analogues have the same effect. We therefore cultured $\mathrm{CD} 14^{+}$monocytes in the presence of rhRANKL and increasing concentrations of PDM02 for 7 days. When compared with cultures treated with rhM-CSF alone, rhRANKL induced the formation of numerous $\mathrm{TRAP}^{+}$ multinucleated cells (Fig. 1a, b), increased the levels of TRAP activity in media (Fig. 1c), and strongly induced the expression of cathepsin K (Fig. 1d), all known to be hallmarks of OC differentiation. In contrast, simultaneous treatment of differentiating cultures with PDM02 significantly reduced the number of multinucleated cells staining for TRAP, in a dose-dependent manner (Fig. 1b). Moreover, PDM02-treated cells showed changes in their morphology, becoming spindle-shaped and resembling the monocytic phenotype observed in wells treated only with rhM-CSF (Fig. 1a) as did RSV-treated cells (Fig. 1a and [10]). Also, the rhRANKL-induced TRAP activity in media was diminished and the observed reduction was dosedependent (Fig. 1c). Similarly, the expression of cathepsin $\mathrm{K}$ was strongly suppressed in cultures treated with PDM02 in a dose-dependent manner (Fig. 1d).

Similar experiments were performed for RSV and the other four analogues listed in Table 1. Results of these experiments are summarized in the table (Fig. 1e), and the corresponding dose-response curves are shown in Supplementary Figs. 1 and 2. In all experiments combined treatment of OC precursors with rhM-CSF and rhRANKL induced a mature OC phenotype. Supplementation of the culture media with either PDM10 or PDM11 reduced the levels of TRAP activity released into the culture media (Supplementary Fig. 1) and suppressed the expression of cathepsin $\mathrm{K}$ in a dose-dependent fashion. For PDM10, the dose-dependent decrease of the number of $\mathrm{TRAP}^{+}$multinucleated cells was also verified (Supplementary Fig. 2). Neither PDM01 nor PDM06 showed any significant inhibition of differentiating OCs (Online Resource 1 in Supplementary material). Note that TRAP slightly increased at the lower concentrations of PDM01, -02, and -10 (not of PDM06 and -11); but this increase was not significant. None of the analogues affected cell viability in this OC differentiation model, as opposed to RSV, which reduced the number of viable cells by $35 \%$ at $100 \mu \mathrm{M}$ (Fig. 1e). Thus, we conclude that PDM02, PDM10, and PDM11 are strong inhibitors of OC differentiation, the former two showing a 1,000-fold higher potency compared to RSV.

Since the primary function of mature OCs is to resorb bone, it was of interest to determine whether RSV analogues could inhibit bone resorption in vitro. $\mathrm{CD} 14^{+}$cells were differentiated with rhM-CSF and rhRANKL as described, and once they became mature OCs, they were seeded on bone slices and exposed to increasing concentrations of PDM02. Figure 2 shows that rhRANKL induced numerous pits yielding $8 \%$ of resorbed bone area. This induction of pits was not affected by PDM02, and neither was cell viability. Similar results were obtained for PDM10 (data not shown).

RSV Analogues Favor the Expression of Monocytic Markers at the Expense of RANK during OC Differentiation

As M-CSF/RANKL-induced osteoclastogenesis was strongly inhibited in the presence of RSV analogues, we tested their effect on the expression of RANK, which is obligatory for inducing OC differentiation, and compared it to the expression of broader myeloid markers. The expression of RANK was significantly reduced in the presence of RSV analogues at the concentration range that inhibited OC differentiation (100 $\mathrm{nM}$ to $1 \mu \mathrm{M})$ compared to cultures with M-CSF/RANKL alone (Fig. 3). In contrast, c-fms gene expression was significantly upregulated in the presence of $100 \mathrm{nM}$ PDM02 or PDM10, a concentration that fully inhibited OC differentiation, whereas at high dose $(1 \mu \mathrm{M})$, this effect was reversed (Fig. 3). On the other hand, PDM11, exhibiting less potent inhibition of OC differentiation (TRAP activity $\mathrm{IC}_{50}=1.2 \mu \mathrm{M}$ ), induced higher expression of the c-fms gene in the range $100 \mathrm{nM}$ to $1 \mu \mathrm{M}$ (Fig. 3).

Similarly to c-fms, expression of the lipopolysacharide receptor CD14 was significantly upregulated by low doses (10 and $100 \mathrm{nM}$ ) of RSV analogues PDM02 and PDM10, whereas high doses $(1 \mu \mathrm{M})$ suppressed CD14 expression. Treatment with PDM11, with less potency toward differentiating OCs, at concentrations ranging from $10 \mathrm{nM}$ to $1 \mu \mathrm{M}$ induced CD14 expression (Fig. 3). We conclude that treatment with RSV analogues induces a similar expression pattern of the monocytic markers c-fms and CD14, which is clearly distinct from the effects on RANK expression. The effects of RSV analogues on these markers parallel those they have on OC generation from monocytes. When 


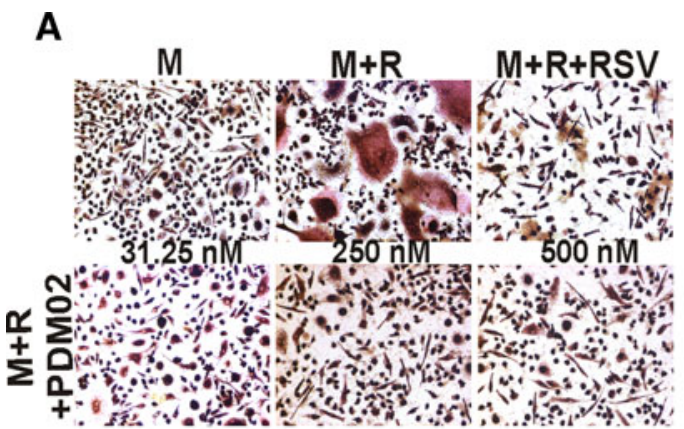

C

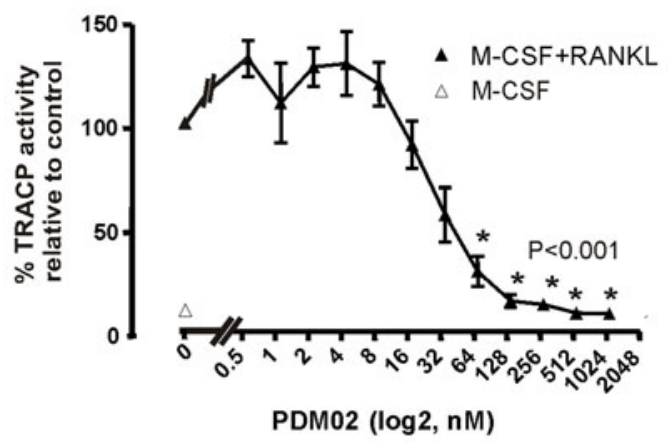

B



D

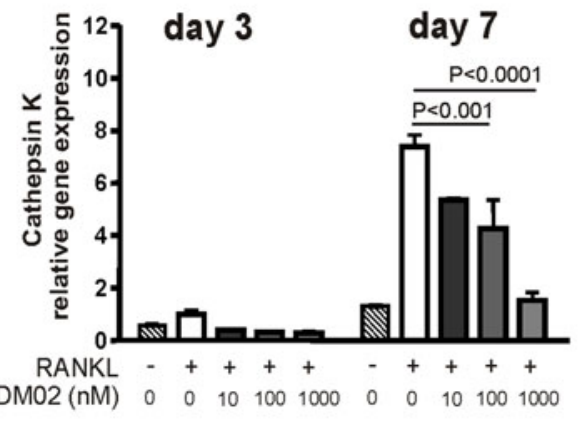

E

The effects of RSV analogues on osteoclast differentiation

\begin{tabular}{|c|c|c|c|c|}
\hline Analogue & $\begin{array}{c}\text { Reduction of TRAP } \\
\text { activity }\end{array}$ & $\begin{array}{c}\text { Inhibition of the number } \\
\text { of multinucleated } \\
\text { TRAP+ cells }\end{array}$ & $\begin{array}{l}\text { Inhibition of } \\
\text { cathepsin K gene } \\
\text { expression to } \\
\text { baseline }\end{array}$ & $\begin{array}{c}\% \text { cell viability upon } \\
\text { treatment with } 100 \\
\mu M \text { of analogue } \\
( \pm S D) \text { stratified to } \\
\text { controls }^{\star \star}\end{array}$ \\
\hline $\begin{array}{l}\text { PDM01 } \\
\text { PDM02 } \\
\text { PDM06 } \\
\text { PDM10 } \\
\text { PDM11 } \\
\text { RSV }\end{array}$ & $\begin{array}{c}\mathrm{EC}_{50}>100 \mu \mathrm{M} \\
\mathrm{EC}_{50}=0.01678 \mu \mathrm{M} \\
\mathrm{EC}_{50}>100 \mu \mathrm{M} \\
\mathrm{EC}_{50}=0.03125 \mu \mathrm{M} \\
\mathrm{EC}_{50}=1.2 \mu \mathrm{M} \\
\mathrm{EC}_{50}=50 \mu \mathrm{M}\end{array}$ & $\begin{array}{c}\text { nd } \\
\mathrm{EC}_{50}=0.03535 \mu \mathrm{M} \\
\text { nd } \\
\mathrm{EC}_{50}=0.06241 \mu \mathrm{M} \\
\text { nd } \\
\mathrm{EC}_{50}=100 \mu \mathrm{M}\end{array}$ & $\begin{array}{c}\text { nd } \\
1 \mu \mathrm{M} \\
\text { nd } \\
100 \mathrm{nM} \\
>1 \mu \mathrm{M} \\
100 \mu \mathrm{M}\end{array}$ & $\begin{array}{c}97.8( \pm 13.9) \\
100( \pm 6.8) \\
115.6( \pm 8.6) \\
115.1( \pm 8.3) \\
116.5( \pm 4.2) \\
65.2( \pm 2.5)\end{array}$ \\
\hline
\end{tabular}

* cultures treated with rhM-CSF alone

** cultures treated with rhM-CSF and rhRANKL

Fig. 1 Effect of RSV analogues on RANKL-induced OC differentiation. Representative images of cell morphology at the end of 7-day cultures after TRAP staining (a). Magnification $\times 20$. PDM02 treatment inhibits the formation of multinucleated $\mathrm{TRAP}^{+}$OCs (b) and the level of TRAP activity (c). Each point represents the mean \pm SD of six cultures. $* P<0.001$, effect of PDM02 compared with cultures in the presence of M-CSF (M) and RANKL (R) alone.

calculating these gene expressions relative to TRAP, the same conclusions are reached (not shown).

RSV Analogues Act on NF- $\kappa$ B Activation and on the NFATc1 Level

Stimulation of RANK during OC differentiation results in $\mathrm{NF}-\kappa \mathrm{B}$ activation followed by induction of NFATc1
PDM02 inhibits expression of cathepsin $\mathrm{K}$ in differentiating OCs (d). Bars represent means \pm SD of three cultures. The table summarizes experiments performed with RSV and other analogues (e). Cathepsin $\mathrm{K}$ expressions are representative of at least two independent experiments. The other results are representative of three independent experiments involving six cultures each. $n d$ not determined

expression. We assessed the effect of RSV analogues on $\mathrm{NF}-\kappa \mathrm{B}$ activation during OC differentiation by assaying the nuclear translocation of its p65 component (Fig. 4). As expected, transient stimulation with rhRANKL increased the number of cells with p65 in the nucleus compared to the cultures treated with rhM-CSF alone (Fig. 4a). Pretreatment for $1 \mathrm{~h}$ or 7 days with PDM02 abrogated the rhRANKL-triggered translocation of p65 to 
Fig. 2 Treatment with PDM02 has no effect on OC bone resorption. Representative images of bone slices exposed to OCs treated with PDM02 $(100 \mu \mathrm{M})$ or not $(\mathbf{a})$.

Magnification $\times 10$. Resorbed bone area was quantified and expressed as a percentage of total bone area (b). Each point represents the mean $\pm \mathrm{SD}$ of six cultures
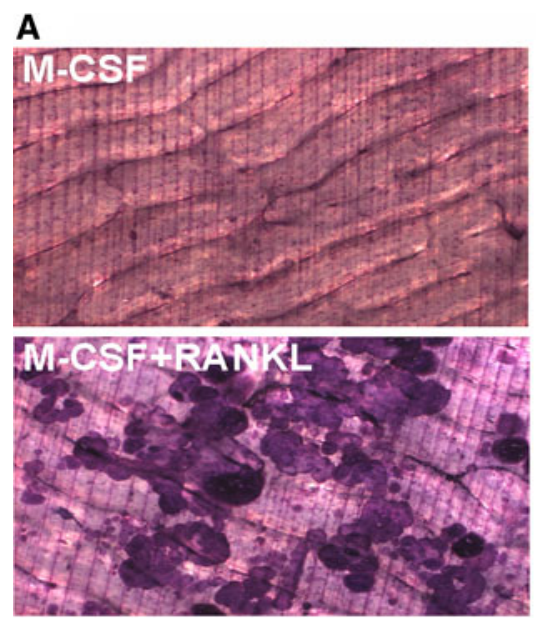

M-CSE+RANKL+PDM02

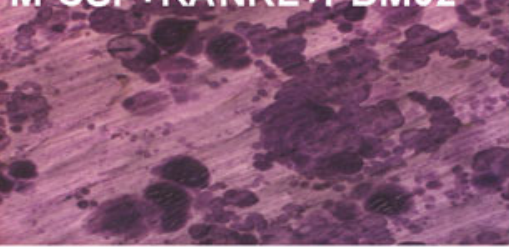

B
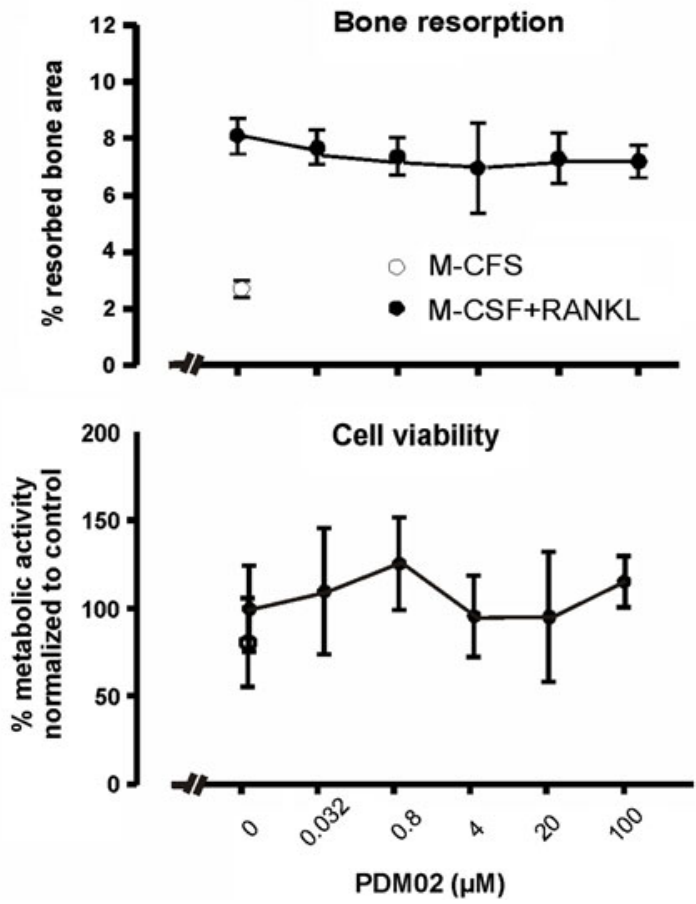

Fig. 3 Effect of RSV analogues on the expression of myeloid markers. Monocytes were cultured for 7 days with M-CSF with (filled square) or without RANKL (filled square) in the presence or absence of increasing concentrations of RSV analogues. Gene expression determined by Q-PCR was normalized to human GUS and ABL, and the relative expression is shown as a percentage compared to control cultures treated with M-CSF and RANKL. Each point is the mean \pm SD of three cultures. Results are representative of two independent experiments. $* P<0.001$
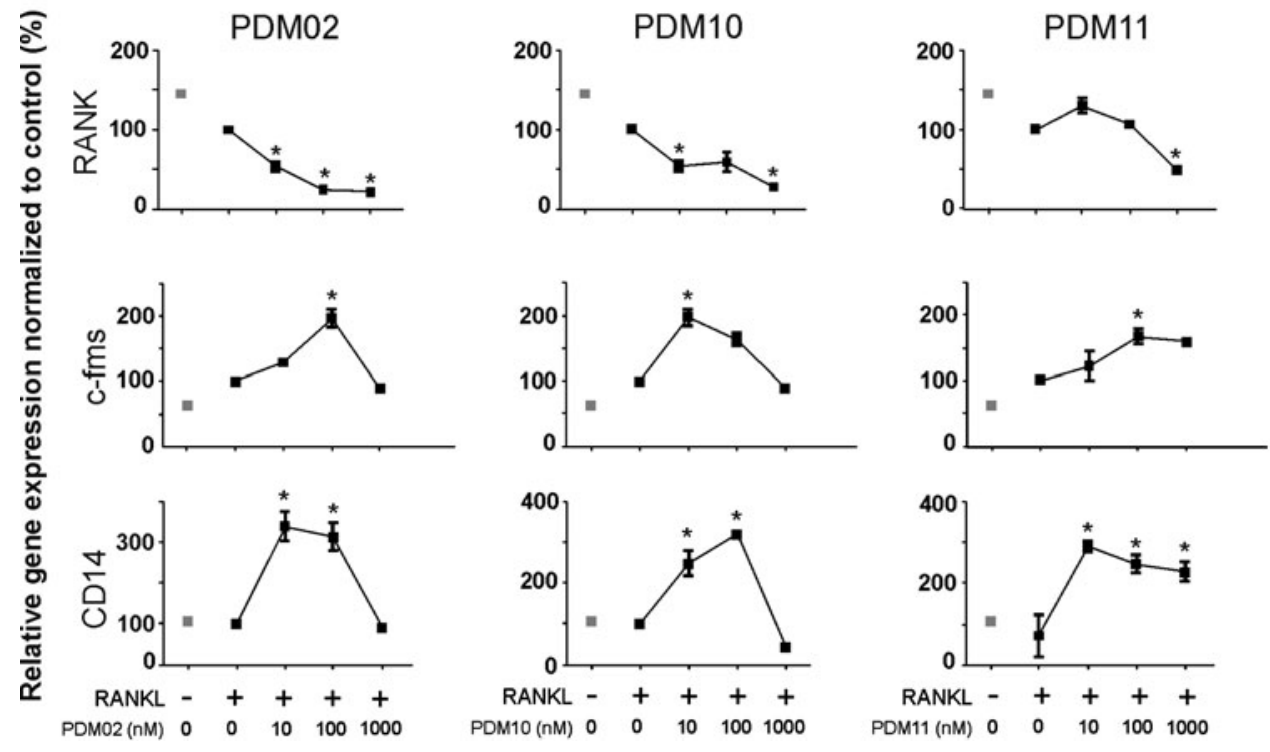

the nucleus and activation of NF- $\kappa \mathrm{B}$ even at low doses (Fig. 4a). Note for comparison that 1-h pretreatment with RSV prior to adding rhRANKL was not sufficient to prevent the nuclear translocation of NF- $\kappa \mathrm{B}$, whereas 7day treatment resulted in an inhibitory effect as previously reported [10].

As expected, rhRANKL increased the expression of NFATc1 compared to control cells cultured with rhM-CSF alone (Fig. 4b). Seven days of treatment with RSV, PDM02, PDM10, or PDM11 at doses that were inhibitory for OC differentiation significantly reduced NFATc1 expression. However, stimulatory activities were obtained at lower doses of these RSV analogues $(10 \mathrm{nM})$ at this 7-day time point, although not after 1 -h treatment. At this early time point, PDM10 and PDM11 already induced a reduction in NFATc1, whereas RSV and PDM02 did 
Fig. 4 Effect of RSV and its analogues on RANKL-mediated NF- $\kappa$ B activation and NFATc1 expression in differentiating OCs. One-hour or 7-day treatment with PDM02 suppressed RANKL-induced nuclear translocation of NF- $\kappa \mathrm{B}$ (a). Total numbers of cells counted are given above in parenthese for each condition. Effect of PDM02, PDM10, PDM11, and RSV $(100 \mu \mathrm{M})$ on NFATc1 gene expression in differentiating OCs after 1 hour or 7-day treatment with analogues and RANKL (b). Each bar is the mean \pm SD value relative to human GUS and $\mathrm{ABL}$ of three cultures. $* P<0.05$, ** $P<0.01$, *** $P<0.001$ statistically significant effect of PDM02 compared to cells cultured without analogues. Results are representative of two independent experiments
A
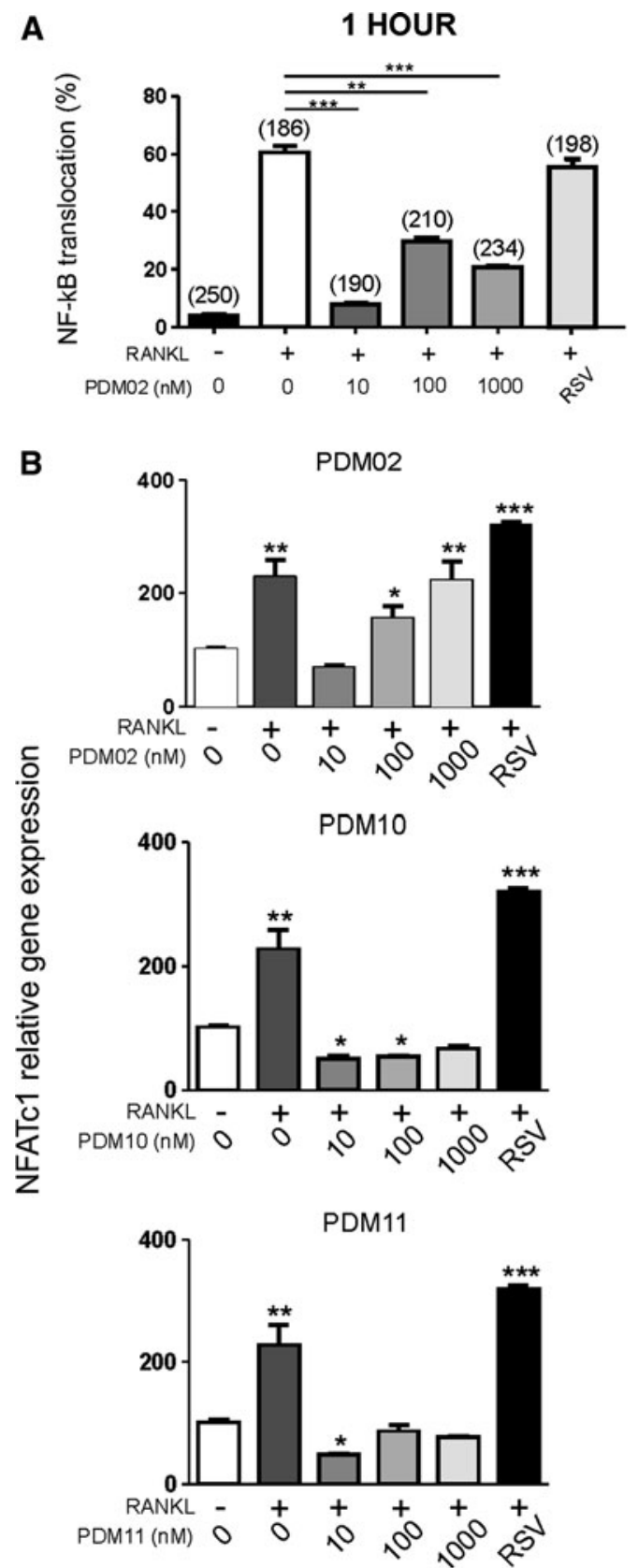

7 DAYS
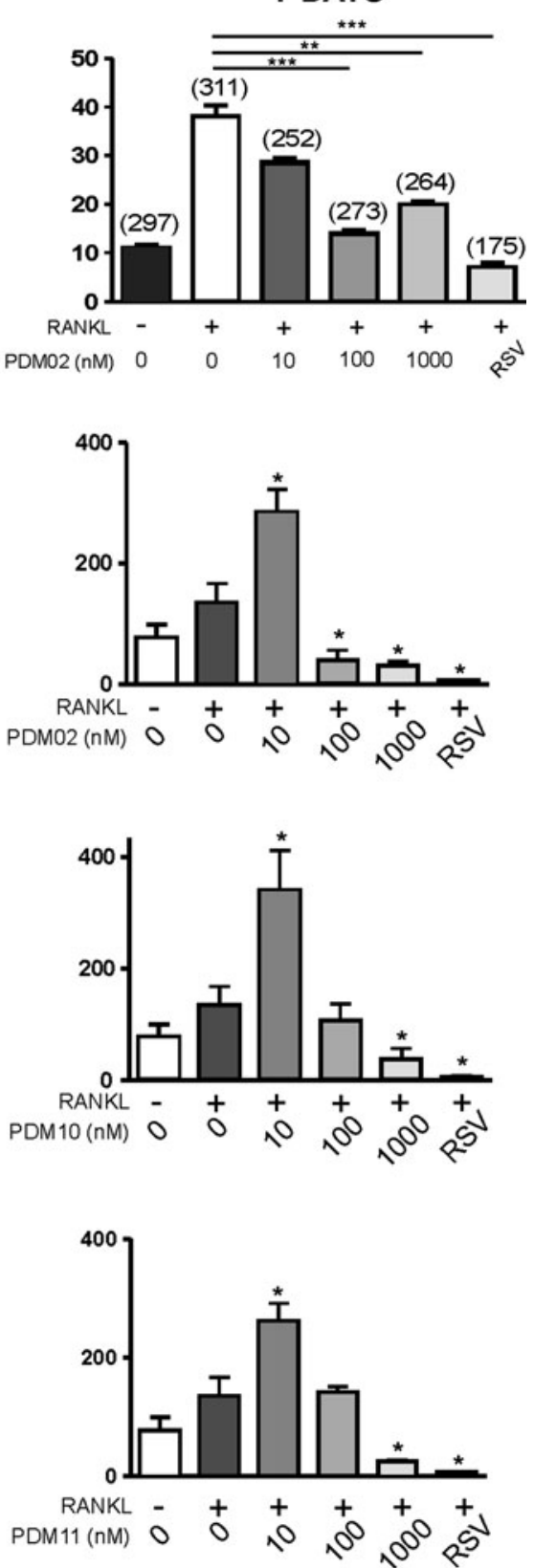

not do so when used at the inhibitory doses for $\mathrm{OC}$ differentiation.

PDM02 Promotes Osteoblast Differentiation from hMSC-TERT Precursors

RSV was previously shown to induce the differentiation of OBs from mesenchymal precursors [10]. Therefore, we evaluated whether RSV analogues also possess osteogenic potential. When culturing hMSC-TERT cells under osteogenic conditions in the presence of increasing concentrations of PDM02 or $100 \mu \mathrm{M}$ RSV, the expression of osteogenic markers such as vitamin $\mathrm{D}_{3}$ receptor (VD3R), osteocalcin, osteopontin (OPN), and alkaline phosphatase (ALP) was upregulated relative to cells not treated with PDM02 or RSV (Fig. 5). Levels of upregulation by PDM02 were comparable to those induced by $100 \mu \mathrm{M}$ RSV at PDM02 doses of $10 \mathrm{nM}$ for ALP, $5 \mu \mathrm{M}$ for VD3R, and $50 \mu \mathrm{M}$ for osteocalcin. Although a twofold upregulation of OPN was already detected at $0.5 \mu \mathrm{M}$ PDM02, higher doses did not cause further upregulation. In contrast, eightfold upregulation was achieved by $100 \mu \mathrm{M}$ RSV. Treatment with PDM02 in the absence of osteogenic factors was inefficient to induce OB maturation (not shown), 
A

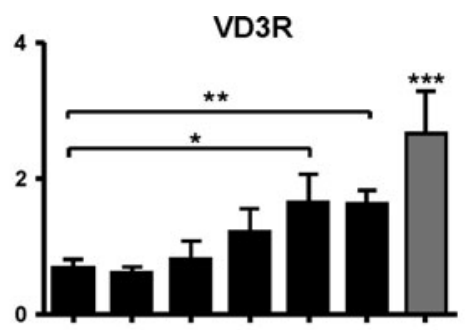

B

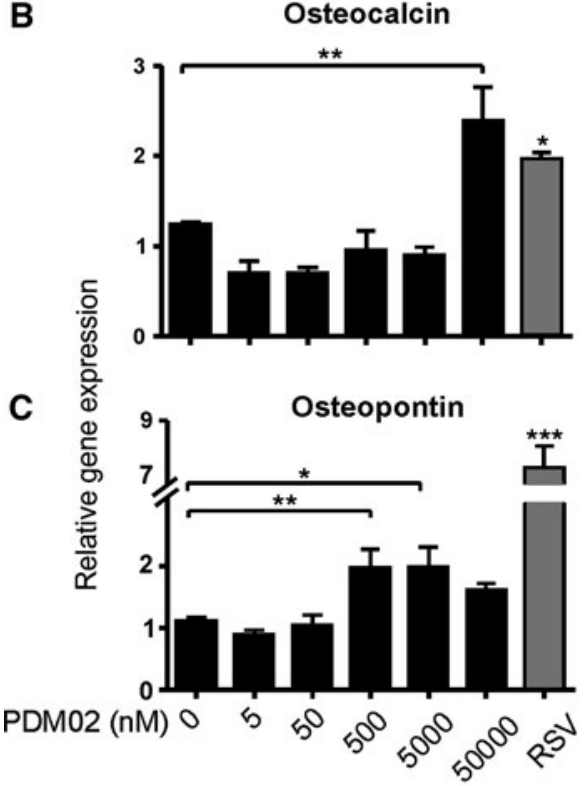

D

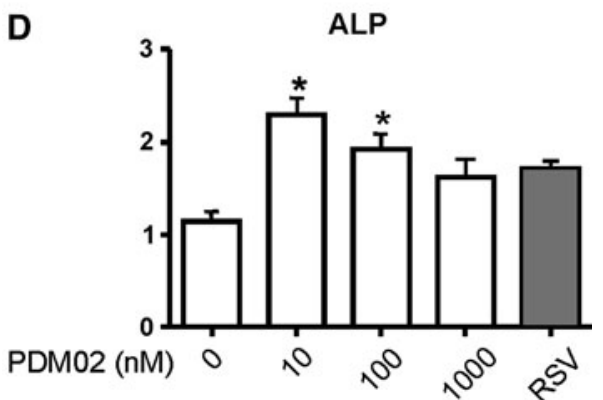

Fig. 5 Effect of PDM02 on the differentiation of hMSC-TERT cells into OBs. hMSC-TERT cells were differentiated into OBs with ODM for $3(\mathbf{a}-\mathbf{c})$ or 5 (d) days in the presence or absence of PDM02 at indicated concentrations (black bars) or $100 \mu \mathrm{M} \mathrm{RSV} \mathrm{(gray} \mathrm{bars).}$ The expression of (a) vitamin D receptor (VDR), (b) osteocalcin, (c) osteopontin, and (d) alkaline phosphatase (ALP) was quantified by real-time PCR and is shown relative to the expression of $\beta$-actin. Each bar is the mean $\pm \mathrm{SD}$ of three independent experiments. $* P<0.01$, ** $P<0.001$ statistically significant effect of PDM02 compared to cells cultured without PDM02. The results are representative of two independent experiments, $* * * P<0.0001$

in contrast with RSV [10]. Cell numbers as evaluated through metabolic activity were not affected by PDM02 in these cultures. Similar experiments were performed for PDM10 and PDM11 without reproducible results (not shown).
RSV Analogues Do Not Show an Effect

on Proliferation and Survival of Myeloma Cells

RSV was previously shown to have a cytotoxic effect on myeloma cells. Therefore, it was of interest to test RSV analogues on myeloma cells. To this end, we treated myeloma cell lines U266 and OPM-2 as well as primary myeloma cells with all RSV analogues listed in Table 1 at doses in the range of $1 \mathrm{nM}$ to $100 \mu \mathrm{M}$ in the presence or absence of serum. Treatment with the RSV analogues did not affect metabolic activity of these cells (data not shown). No signs of apoptosis were observed in AnnexinV-stained cells (data not shown). We concluded that these RSV analogues have no effect on myeloma cell survival.

PDM02 Does Not Show a Significant Effect on OVX-Induced Bone Loss

In vitro screening identified PDM02 as the most potent RSV analogue of those listed in Table 1, strongly inhibiting OC differentiation at a nanomolar concentration without nonspecific toxicity and promoting OB maturation. In order to evaluate the in vivo relevance of these effects, we tested PDM02 in OVX-induced osteoporosis in rats. To this end, OVX animals were treated with either high $(40 \mathrm{mg} / \mathrm{kg})$ or low $(4 \mathrm{mg} / \mathrm{kg})$ doses of PDM02 for 6 weeks. As expected, OVX significantly reduced total BMD compared with shamoperated animals, and this effect was partially prevented by administration of estrogen (Fig. 6). Administration of vehicle alone induced a further decline in BMD compared with untreated OVX controls. PDM02 had no statistically significant effect on BMD or bone area (whether total or trabecular) or CTX levels (whether at the 2- or 6-week time point) in OVX rats (Fig. 6 and data not shown).

\section{Discussion}

RSV's ability to simultaneously target the three critical cell players in multiple myeloma-induced bone disease (OCs, OBs, and myeloma cells) made it a very attractive potential therapeutic agent with possible relevance to other bone pathologies. However, its limited bioavailability by oral administration $[18,19]$ and too broad spectrum of activities and undesired side effects $[2,3,20]$ stimulated a search for analogues that had preserved the therapeutic effect but were without the side effects of the natural product. Our study identified two compounds, PDM02 and PDM10, which are at least 1,000 times more potent in terms of inhibition of OC differentiation compared to RSV. Since OCs have a short half-life, it may be anticipated that exposure to these RSV analogues will also result in strong inhibition of bone resorption, even if in vitro these 
Fig. 6 PDM02 does not show a significant effect in an ovariectomy model of osteoporosis. Rats were either sham-operated (SHAM) or ovariectomized (OVX) and left untreated $(-)$ or treated for 6 weeks with estrogen $(\mathrm{E})$, vehicle $(\mathrm{V})$, or PDM02 at a dose of 40 or $40 \mathrm{mg} / \mathrm{kg}$. BMD and total cross-sectional area at the tibial metaphysis were measured as described in "Materials and methods". Each point represents sample measurement in respective populations ( $n=10$ /group). $* P<0.01, * * * P<0.0001$
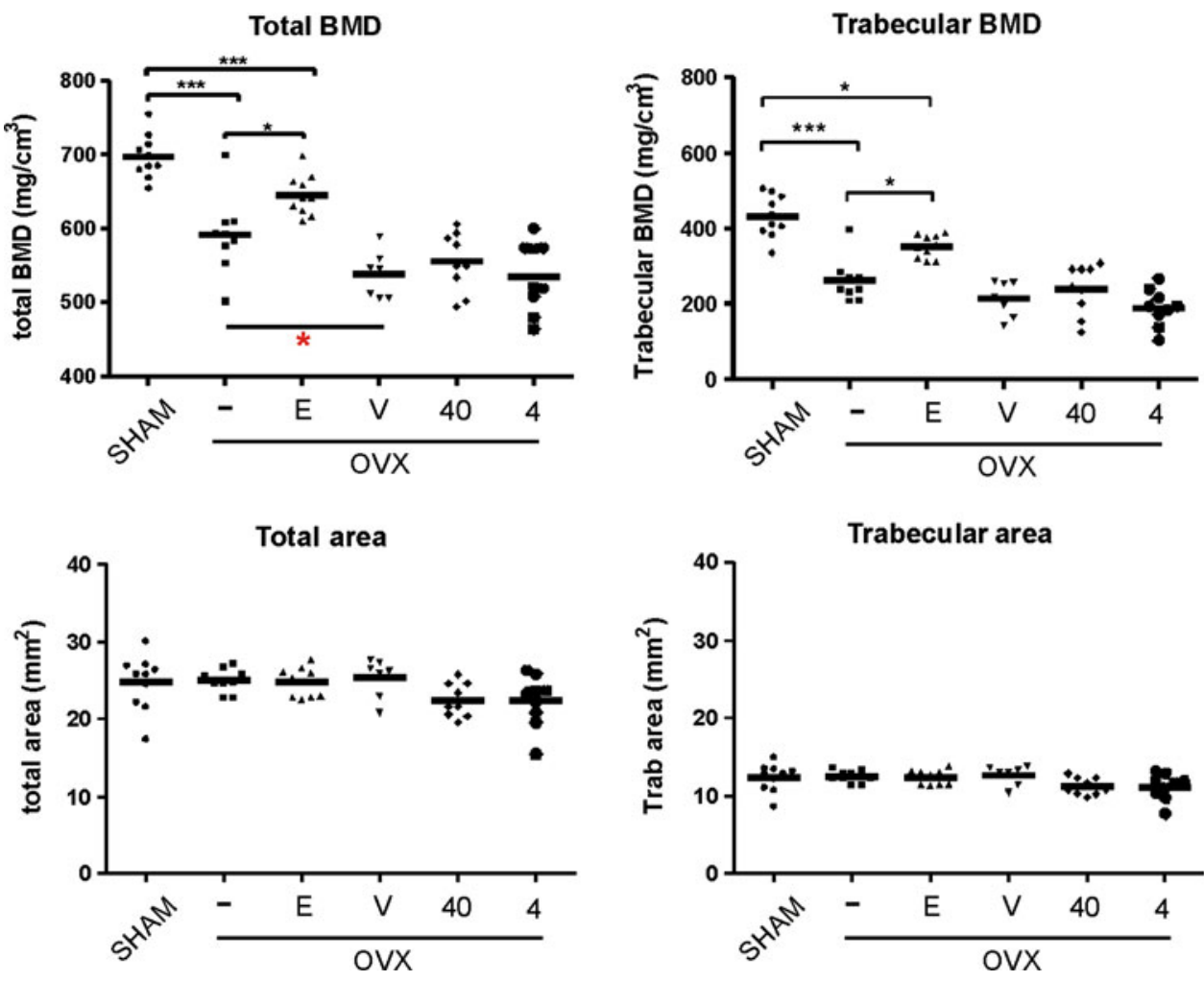

Trabecular area

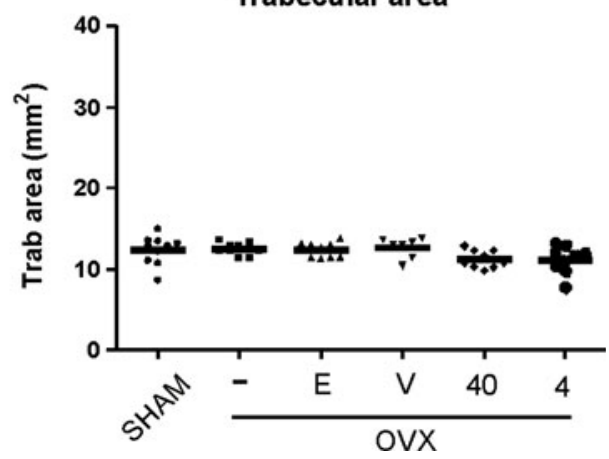

compounds proved to be without direct effect on the bone resorption process itself. Furthermore, PDM02 stimulated OB maturation at 100 times lower concentrations than RSV. In contrast to RSV, these analogues were inactive against myeloma cells, and PDM02 could not protect against OVX-induced bone loss.

The strong effects of PDM02, PDM10, and, to a lesser extent, PDM11 on OC differentiation were demonstrated in three different ways: (1) by inhibition of the formation of TRAP-positive multinucleated cells, (2) by inhibition of the release of TRAP in the medium, and (3) by downregulation of the expression of cathepsin $\mathrm{K}$, all widely recognized hallmarks of OC differentiation.

The mechanism mediating their effect is not merely broad cytotoxicity since the most effective compound PDM02 acted at a dose 5,000 lower than RSV without affecting cell viability, as was the case for the active dose of RSV. Another indication of lack of cytotoxicity is the absence of a direct effect on OC resorptive activity, as mentioned above.

An interesting feature of RSV analogues compared to RSV is the lack of affinity to the ER. Several studies have previously shown that estrogen could directly inhibit OC differentiation [37]. Therefore, it was plausible that the effects of RSV on OCs seen in the present and previous study [10] were ER-mediated. The present study rules out this hypothesis since it shows that RSV derivatives which do not bind the ER still inhibit OC differentiation.
Interestingly, however, even if RSV analogues differ from RSV by lower cytotoxicity and lack of ER binding affinity, they share with RSV the ability to target the key molecules controlling OC differentiation. Indeed, both RSV and its analogues interfere with M-CSF/RANKL signaling and inhibit activation of NF- $\kappa$ B and NFATc1. Treatment with RSV [10] and RSV analogues induced the expression of c-fms while downregulating the expression of RANK, thus retaining the monocytic phenotype of OC progenitors. In agreement with this, CD14 is upregulated and the monocytic spindle-like morphology was observed for PDM02-treated cells, two features which were also observed in the presence of RSV [10]. NF- $\kappa$ B and NFATc1 function downstream of RANKL signaling and play an essential role in the developmental stage from monocyte precursors to mature OCs. We showed that short treatment with RSV analogues (30 minutes after exposure to RANKL), even at $10 \mathrm{nM}$, significantly diminished nuclear translocation of NF- $\kappa \mathrm{B}$ required for RANKL signaling. At the culture time where control cells differentiate into OCs and show high NFATc1 levels, the RSV analogues at concentrations between $100 \mathrm{nM}$ to $1 \mu \mathrm{M}$ reduce both OC differentiation and NFATc1 expression. Noteworthy, at the same time point, a lower concentration $(10 \mathrm{nM})$ of PDM02, PDM10, and PDM11 stimulated NFATc1; and in the case of the former 2 analogues, this stimulation correlated with a trend toward increased OC differentiation. This correlation did not hold true for PDM11, however, thereby 
showing that there is no general mechanism by which RSV analogues at low concentration tend to stimulate OC differentiation. Interestingly also, NFATc 1 and NF- $\kappa \mathrm{B}$ do not show an identical inhibition pattern. This difference reflects the fact that NFATc1 is regulated not only by NF- $\kappa \mathrm{B}$ but also by the c-Fos and the $\mathrm{Ca}$ /calcineurin pathway, as well as by NFATc1-mediated activation of its own promoter resulting in autoamplification [38]. NFATc1 is thus downstream of several "signaling modules" and integrates them, whereas NF- $\kappa \mathrm{B}$ activity is known to rise and fall rapidly, acting as an early immediate signal [39].

In relation to RSV analogues as AhR ligands, it is of interest to mention that NFATc1 was reported to be activated upon the engagement of AhR in the breast cancer cell line MCF-7 [40]. Furthermore, NF- $\kappa \mathrm{B}$ is also triggered when AhR is activated so that $\mathrm{NF}-\kappa \mathrm{B}$ acts as a common transcriptional factor for both RANKL and AhR-mediated signaling. Recent results suggested a competitive model of RANKL/AhR activation [41]. The AhR ligand was shown to directly inhibit NF- $\kappa \mathrm{B}$ activation and thus osteoclastogenesis. This effect was reversed by high concentrations of RANK and by RSV with antagonistic properties toward AhR [41]. Although these observations stress a close relation between the AhR, which is known to be a target for RSV analogues, and NF- $\kappa \mathrm{B} / \mathrm{NFATc} 1$, which is shown here to respond to the RSV analogues, they do not allow a full understanding of our data. Indeed, PDM02 and PDM11 are AhR antagonists, while PDM10 is an agonist (Table 1); but they all displayed the same inhibitory effect toward OC differentiation, and furthermore, RSV always showed inhibitory activity ([10] and present results), in contrast with the situation of Voronov et al. [42], where it was tested in the presence of AhR agonists and stimulated resorption.

A characteristic of the extensive bone loss observed in osteoporotic and some cancer patients is that increased bone resorption is not compensated by enhanced bone formation [43]. Formation of new bone is mediated by OBs that differentiate from mesenchymal stem cells in response to different hormones or local factors. Previous studies showed that RSV induced the mature phenotype in OB precursors [11] while decreasing adipocyte formation from mesenchymal precursors in favor of osteogenesis [44]. In our study we demonstrated that only PDM02 was able to promote the mature phenotype in hMSC-TERT. PDM02 induced the expression of VDR, OPN, osteocalcin, and ALP as important characteristics of $\mathrm{OB}$ differentiation. RSV analogues used in the study displayed different binding affinities to AhR and acted either as antagonists or as agonists.

The occupancy of AhR by dioxins has been correlated with suppression of OB maturation [33]. This dioxininduced suppression could be reduced by simultaneous treatment with RSV, acting strongly antagonistically on
AhR [12]. PDM02 is a 140 times more potent antagonist of AhR than RSV and correspondingly affected OB differentiation at lower concentrations compared to RSV. However, treatment of MSC-TERT cells with PDM10 and PDM11-respectively, an AhR agonist and antagonistdid not give reproducible results. Therefore, the stimulatory properties of PDM02 on OBs cannot be clearly interpreted as being mediated through AhR.

In multiple myeloma, cancer cells promote OC activation and induce bone resorption [45-47] while suppressing bone formation. We previously demonstrated cytotoxic and proapoptotic properties of RSV on myeloma cells [10] on a molecular basis explained by inhibition of the constitutional activation of $\mathrm{NF}-\kappa \mathrm{B}$ in myeloma cells [15]. In the present study we tested several RSV analogues and none of them showed antagonistic activity against either primary myeloma cells or myeloma cell lines. This discrepancy could be related to the fact that the tested analogues are structurally modified and dihydroxyl groups in RSV were replaced. Indeed, several recent studies with RSV and its natural or synthetic analogues underscore the importance of 3,4-dihydroxyl groups in exhibiting cytotoxic and proapoptotic activities [48]. Furthermore, Murias and colleagues [24] clearly demonstrated that hydroxyl groups are critical and that the natural RSV analogue piceatannol containing four hydroxyl groups exhibited more cytotoxicity toward leukemia cells than the trihydroxystilbene RSV. Accordingly, compounds lacking hydroxyl groups such as rhaponticin and stilbene performed poorer than RSV and piceatannol, confirming the importance of hydroxyl groups [23].

Among five analogues, only PDM02 both inhibited the differentiation of OCs and induced $\mathrm{OB}$ maturation in a nanomolar range, although it could not antagonize myeloma cells. Based on these observations, we tested the efficacy of PDM02 in vivo, using OVX rats as a model of human postmenopausal osteoporosis. It was reported that low-dose RSV given by force-feeding for 12 weeks had a beneficial effect on BMD and bone calcium content in OVX rats [49], despite its low bioavailability. In our study, the RSV analogue PDM02 administered intraperitoneally for 6 weeks had no significant effect on the loss of BMD or on serum CTX, which is a marker of bone resorption. The differences between our results and those of Liu et al. [49] may be due to the duration of the experiment (6 vs. 12 weeks), to the nature of the drug itself, or to different drug-administration modes (intraperitroneal injection vs. gavage). In our system, PDM02 was dissolved in DMSO, and administration of DMSO alone induced further bone loss compared with untreated OVX animals. It cannot be ruled out that DMSO dosing provoked inflammation and that the release of inflammatory cytokines caused further bone loss [reviewed in 50]. Thus, the lack of bone-sparing 
effects of PDM02 in vivo could be a result of DMSO administration. Regarding the drug itself, one may also speculate that the lack of in vivo effect of PDM02 may be due to low bioavailability. The bioavailability of PDM02 is unknown. Injected PDM02 could be rapidly excreted from the body, as is the case for RSV. A recent study suggested that RSV is mainly absorbed in the small intestine as RSV glucuronide [19]. Glucuronides of phenolic compounds are thought to be rapidly excreted in vivo and to be pharmacologically inactive [51]. PDM02 could be processed in the same or a similar way, thereby rapidly losing the activity demonstrated in vitro or maybe falling in the low concentration range where a trend to higher TRAP and NFATc1 was detected in culture conditions. Lack of efficacy might also be explained by the fact that PDM02's inhibitory activity is restricted to $\mathrm{OC}$ differentiation, whereas strong antiresorptives such as bisphosphonates and denosumab not only inhibit OC differentiation but also directly inhibit OC bone resorption.

To our knowledge, the present study is the first to show that RSV analogues strongly inhibit OC differentiation and may promote OB differentiation. Therefore, RSV analogues may be of potential therapeutic interest. However, further studies are required in order to define their pharmacological characteristics and pharmacodynamics with the aim to achieve in vivo efficacy.

Acknowledgments The authors thank Birgit MacDonald and Christiane Schüler for excellent technical assistance. This work was supported by grants from the Region of Southern Denmark.

Open Access This article is distributed under the terms of the Creative Commons Attribution Noncommercial License which permits any noncommercial use, distribution, and reproduction in any medium, provided the original author(s) and source are credited.

\section{References}

1. Jang M, Cai L, Udeani GO, Slowing KV, Thomas CF, Beecher CW, Fong HH, Farnsworth NR, Kinghorn AD, Mehta RG, Moon RC, Pezzuto JM (1997) Cancer chemopreventive activity of resveratrol, a natural product derived from grapes. Science 275:218-220

2. Dong Z (2003) Molecular mechanism of the chemopreventive effect of resveratrol. Mutat Res 523-524:145-150

3. Pervaiz S (2003) Resveratrol: from grapevines to mammalian biology. FASEB J 17:1975-1985

4. Banerjee S, Bueso-Ramos C, Aggarwal BB (2002) Suppression of 7,12-dimethylbenz(a)anthracene-induced mammary carcinogenesis in rats by resveratrol: role of nuclear factor-kappaB, cyclooxygenase 2, and matrix metalloprotease 9. Cancer Res 62:4945-4954

5. Yu L, Sun ZJ, Wu SL, Pan CE (2003) Effect of resveratrol on cell cycle proteins in murine transplantable liver cancer. World $\mathbf{J}$ Gastroenterol 9:2341-2343
6. Schneider Y, Duranton B, Gosse F, Schleiffer R, Seiler N, Raul F (2001) Resveratrol inhibits intestinal tumorigenesis and modulates host-defense-related gene expression in an animal model of human familial adenomatous polyposis. Nutr Cancer 39:102-107

7. Tessitore L, Davit A, Sarotto I, Caderni G (2000) Resveratrol depresses the growth of colorectal aberrant crypt foci by affecting bax and p21(CIP) expression. Carcinogenesis 21:1619-1622

8. Kimura Y, Okuda H (2001) Resveratrol isolated from Polygonum cuspidatum root prevents tumor growth and metastasis to lung and tumor-induced neovascularization in Lewis lung carcinomabearing mice. J Nutr 131:1844-1849

9. Chen Y, Tseng SH, Lai HS, Chen WJ (2004) Resveratrol-induced cellular apoptosis and cell cycle arrest in neuroblastoma cells and antitumor effects on neuroblastoma in mice. Surgery 136:57-66

10. Boissy P, Andersen TL, Abdallah BM, Kassem M, Plesner T, Delaisse JM (2005) Resveratrol inhibits myeloma cell growth, prevents osteoclast formation, and promotes osteoblast differentiation. Cancer Res 65:9943-9952

11. Mizutani K, Ikeda K, Kawai Y, Yamori Y (1998) Resveratrol stimulates the proliferation and differentiation of osteoblastic MC3T3-E1 cells. Biochem Biophys Res Commun 253:859-863

12. Singh SU, Casper RF, Fritz PC, Sukhu B, Ganss B, Girard B Jr, Savouret JF, Tenenbaum HC (2000) Inhibition of dioxin effects on bone formation in vitro by a newly described aryl hydrocarbon receptor antagonist, resveratrol. J Endocrinol 167:183-195

13. Ulsperger E, Hamilton G, Raderer M, Baumgartner G, Hejna M, Hoffmann O, Mallinger R (1999) Resveratrol pretreatment desensitizes AHTO-7 human osteoblasts to growth stimulation in response to carcinoma cell supernatants. Int J Oncol 15:955-959

14. Clerc D, Fermand JP, Mariette X (2003) Treatment of multiple myeloma. Joint Bone Spine 70:175-186

15. Sun C, Hu Y, Liu X, Wu T, Wang Y, He W, Wei W (2006) Resveratrol downregulates the constitutional activation of nuclear factor-kappaB in multiple myeloma cells, leading to suppression of proliferation and invasion, arrest of cell cycle, and induction of apoptosis. Cancer Genet Cytogenet 165:9-19

16. Sun CY, Hu Y, Guo T, Wang HF, Zhang XP, He WJ, Tan $\mathrm{H}$ (2006) Resveratrol as a novel agent for treatment of multiple myeloma with matrix metalloproteinase inhibitory activity. Acta Pharmacol Sin 27:1447-1452

17. Anderson KC, Shaughnessy JD Jr, Barlogie B, Harousseau JL, Roodman GD (2002) Multiple myeloma. Hematology Am Soc Hematol Educ Program 1:214-240

18. Goldberg DM, Yan J, Soleas GJ (2003) Absorption of three winerelated polyphenols in three different matrices by healthy subjects. Clin Biochem 36:79-87

19. Marier JF, Vachon P, Gritsas A, Zhang J, Moreau JP, Ducharme MP (2002) Metabolism and disposition of resveratrol in rats: extent of absorption, glucuronidation, and enterohepatic recirculation evidenced by a linked-rat model. J Pharmacol Exp Ther 302:369-373

20. Crowell JA, Korytko PJ, Morrissey RL, Booth TD, Levine BS (2004) Resveratrol-associated renal toxicity. Toxicol Sci 82:614-619

21. Bowers JL, Tyulmenkov VV, Jernigan SC, Klinge CM (2000) Resveratrol acts as a mixed agonist/antagonist for estrogen receptors alpha and beta. Endocrinology 141:3657-3667

22. Stopper H, Schmitt E, Kobras K (2005) Genotoxicity of phytoestrogens. Mutat Res 574:139-155

23. Ashikawa K, Majumdar S, Banerjee S, Bharti AC, Shishodia S, Aggarwal BB (2002) Piceatannol inhibits TNF-induced NFkappaB activation and NF-kappaB-mediated gene expression through suppression of IkappaBalpha kinase and p65 phosphorylation. J Immunol 169:6490-6497

24. Murias M, Jager W, Handler N, Erker T, Horvath Z, Szekeres T, Nohl H, Gille L (2005) Antioxidant, prooxidant and cytotoxic 
activity of hydroxylated resveratrol analogues: structure-activity relationship. Biochem Pharmacol 69:903-912

25. Mundy GR, Edwards JR, Zainabadi K, Elefteriou F, Alt F, Guarente L (2008) The aging associated gene SIRT-1 controls bone mass in vivo through osteoblast and osteoclast regulation. Bone S49

26. de Medina P, Casper R, Savouret JF, Poirot M (2005) Synthesis and biological properties of new stilbene derivatives of resveratrol as new selective aryl hydrocarbon modulators. J Med Chem 48:287-291

27. Shiau AK, Barstad D, Loria PM, Cheng L, Kushner PJ, Agard DA, Greene GL (1998) The structural basis of estrogen receptor/ coactivator recognition and the antagonism of this interaction by tamoxifen. Cell 95:927-937

28. Zallone A (2006) Direct and indirect estrogen actions on osteoblasts and osteoclasts. Ann N Y Acad Sci 1068:173-179

29. Dertinger SD, Nazarenko DA, Silverstone AE, Gasiewicz TA (2001) Aryl hydrocarbon receptor signaling plays a significant role in mediating benzo $[a]$ pyrene- and cigarette smoke condensateinduced cytogenetic damage in vivo. Carcinogenesis 22:171-177

30. Mandal PK (2005) Dioxin: a review of its environmental effects and its aryl hydrocarbon receptor biology. J Comp Physiol B 175:221-230

31. Marlowe JL, Puga A (2005) Aryl hydrocarbon receptor, cell cycle regulation, toxicity, and tumorigenesis. $\mathrm{J}$ Cell Biochem 96:1174-1184

32. Bock KW (1994) Aryl hydrocarbon or dioxin receptor: biologic and toxic responses. Rev Physiol Biochem Pharmacol 125:1-42

33. Ryan EP, Holz JD, Mulcahey M, Sheu TJ, Gasiewicz TA, Puzas JE (2007) Environmental toxicants may modulate osteoblast differentiation by a mechanism involving the aryl hydrocarbon receptor. J Bone Miner Res 22:1571-1580

34. Abdallah BM, Haack-Sorensen M, Burns JS, Elsnab B, Jakob F, Hokland P, Kassem M (2005) Maintenance of differentiation potential of human bone marrow mesenchymal stem cells immortalized by human telomerase reverse transcriptase gene despite extensive proliferation. Biochem Biophys Res Commun 326:527-538

35. Simonsen JL, Rosada C, Serakinci N, Justesen J, Stenderup K, Rattan SI, Jensen TG, Kassem M (2002) Telomerase expression extends the proliferative life-span and maintains the osteogenic potential of human bone marrow stromal cells. Nat Biotechnol 20:592-596

36. Abdallah BM, Jensen $\mathrm{CH}$, Gutierrez $\mathrm{G}$, Leslie RG, Jensen TG, Kassem M (2004) Regulation of human skeletal stem cells differentiation by Dlk1/Pref-1. J Bone Miner Res 19:841-852

37. Robinson LJ, Yaroslavskiy BB, Griswold RD, Zadorozny EV, Guo L, Tourkova IL, Blair HC (2009) Estrogen inhibits RANKLstimulated osteoclastic differentiation of human monocytes through estrogen and RANKL-regulated interaction of estrogen receptor-alpha with BCAR1 and Traf6. Exp Cell Res 315:1287-1301
38. Shinohara M, Takayanagi H (2007) Novel osteoclast signaling mechanisms. Curr Osteoporos Rep 5:67-72

39. Ferreiro DU, Komives EA (2010) Molecular mechanisms of system control of NF-kappaB signaling by IkappaBalpha. Biochemistry 49:1560-1567

40. Seifert A, Rau S, Kullertz G, Fischer B, Santos AN (2009) TCDD induces cell migration via NFATc1/ATX-signaling in MCF-7 cells. Toxicol Lett 184:26-32

41. Voronov I, Li K, Tenenbaum HC, Manolson MF (2008) Benzo[a]pyrene inhibits osteoclastogenesis by affecting RANKLinduced activation of NF-kappaB. Biochem Pharmacol 75: 2034-2044

42. Voronov I, Heersche JN, Casper RF, Tenenbaum HC, Manolson MF (2005) Inhibition of osteoclast differentiation by polycyclic aryl hydrocarbons is dependent on cell density and RANKL concentration. Biochem Pharmacol 70:300-307

43. Seeman E, Delmas PD (2006) Bone quality - the material and structural basis of bone strength and fragility. N Engl J Med 354:2250-2261

44. Backesjo CM, Li Y, Lindgren U, Haldosen LA (2006) Activation of Sirt1 decreases adipocyte formation during osteoblast differentiation of mesenchymal stem cells. J Bone Miner Res 21:993-1002

45. El Hajj Dib IE, Melanie G, Valery S, Romuald M, Michel B, Kamel S (2008) Multiple myeloma cells directly stimulate bone resorption in vitro by down-regulating mature osteoclast apoptosis. Leuk Res 32:1279-1287

46. Hecht M, von Metzler I, Sack K, Kaiser M, Sezer O (2008) Interactions of myeloma cells with osteoclasts promote tumour expansion and bone degradation through activation of a complex signalling network and upregulation of cathepsin $\mathrm{K}$, matrix metalloproteinases (MMPs) and urokinase plasminogen activator (uPA). Exp Cell Res 314:1082-1093

47. Yaccoby S, Pearse RN, Johnson CL, Barlogie B, Choi Y, Epstein J (2002) Myeloma interacts with the bone marrow microenvironment to induce osteoclastogenesis and is dependent on osteoclast activity. Br J Haematol 116:278-290

48. Cai YJ, Wei QY, Fang JG, Yang L, Liu ZL, Wyche JH, Han Z (2004) The 3,4-dihydroxyl groups are important for trans-resveratrol analogs to exhibit enhanced antioxidant and apoptotic activities. Anticancer Res 24:999-1002

49. Liu ZP, Li WX, Yu B, Huang J, Sun J, Huo JS, Liu CX (2005) Effects of trans-resveratrol from Polygonum cuspidatum on bone loss using the ovariectomized rat model. J Med Food 8:14-19

50. Walsh NC, Crotti TN, Goldring SR, Gravallese EM (2005) Rheumatic diseases: the effects of inflammation on bone. Immunol Rev 208:228-251

51. Kuhnle G, Spencer JP, Chowrimootoo G, Schroeter H, Debnam ES, Srai SK, Rice-Evans C, Hahn U (2000) Resveratrol is absorbed in the small intestine as resveratrol glucuronide. Biochem Biophys Res Commun 272:212-217 